\title{
Fototerapia (LEDs 660/890nm) no tratamento de úlceras de perna em pacientes diabéticos: estudo de caso
}

\author{
Phototherapy (LEDs 660/890nm) in the treatment of leg ulcers in diabetic \\ patients: case study
}

\author{
Débora Garbin Minatel ${ }^{1}$ \\ Suzelei Castro França ${ }^{3}$
}

\author{
Chukuka Samuel Enwemeka² \\ Marco Andrey Cipriani Frade ${ }^{4}$
}

\begin{abstract}
Resumo: Avaliou-se a fototerapia na cicatrização de úlceras de perna (UP) mistas em dois pacientes diabéticos (tipo 2), hipertensos. O aparelho apresentava sonda 1 (S1) (1 LED de 660nm, 5mW) aplicado em 3 UP e sonda 2 (S2) (32 LEDs de 890nm e 4 LEDs de 660nm, 500mW) em 6 UP. Após antissepsia, úlceras foram tratadas com sondas a $3 \mathrm{~J} / \mathrm{cm} 2,30 \mathrm{seg}, 2 \mathrm{x} / \mathrm{semana}$ seguido pelo curativo diário com sulfadiazina de prata a $1 \%$ por 12 semanas. Pela análise com software Image J®, as UP com S2 tiveram índices de cicatrização médios de 0,6 ; 0,7 e 0,9 enquanto $S 1$ foi de 0,$2 ; 0,4$ e 0,6 no $30^{\circ}, 60^{\circ}$ e $90^{\circ}$ dias, respectivamente. A fototerapia acelerou a cicatrização das úlceras de perna em pacientes diabéticos.

Palavras-chave: Cicatrização de feridas; Diabetes Mellitus; Fototerapia; Terapia a laser de baixa intensidade; Úlcera da perna

Abstract: This study evaluated the use of phototherapy in the healing of mixed leg ulcers in two diabetic patients (type 2) with arterial hypertension. The device had probe 1 (one $660 \mathrm{~nm}$ LED, $5 \mathrm{~mW}$ ) applied in 3 ulcers and probe 2 (32 890nm LEDs associated with 4 660nm LEDs, 500mW) in 6 ulcers. After asepsis, ulcers were treated with probes to $3 \mathrm{~J} / \mathrm{cm} 2,30$ sec per point, twice a week, followed by topical daily dressing with $1 \%$ silver sulphadiazine during 12 weeks. The following analyses of ulcers with software Image $\mathrm{J}$ showed that probe 2 presented mean healing rates of $0.6 ; 0.7$ and 0.9 , whereas probe 1 had 0.2 ; 0.4 and 0.6 at 30, 60 and 90 days, respectively. Phototherapy accelerated wound healing of leg ulcers in diabetic patients.

Keywords: Diabetes mellitus; Laser therapy, low-level; Leg ulcer; Phototherapy; Wound healing
\end{abstract}

\section{INTRODUÇÃO}

Diabetes mellitus é causada por desordem metabólica na secreção e/ou ação da insulina, pela hiperglicemia e/ou hiperinsulinemia. ${ }^{1} \mathrm{O}$ diabetes tipo 2 caracteriza-se pela hiperglicemia crônica relacionada à resistência das células alvos à ação da insulina circulante, que leva a desordens degenerativas, devido a macro e/ou microangiopatia e neuropatia, fatores esses que favorecem o surgimento das úlceras de perna e que retardam a apresentam cicatrização das mesmas, justificando a busca de novas condutas terapêuticas.

Nos últimos anos, a fototerapia por luzes coerentes (laseres) e não coerentes (LEDs-Light Emiting Diodes) destaca-se como método bioestimulador para o reparo tecidual,2,3 que aumenta a circulação local, proliferação celular e síntese de colágeno. ${ }^{4,5}$ Vários

\footnotetext{
Recebido em 08.04.2008.

Aprovado pelo Conselho Consultivo e aceito para publicação em 26.01.09.

* Trabalho realizado no Ambulatório de Úlceras da Dermatologia do Centro Saúde Escola da Faculdade de Medicina de Ribeirão Preto (FMRP-USP) - Ribeirão Preto (SP), Brasil.

Conflito de interesse: Nenhum / Conflict of interest: None

Suporte financeiro / Financial funding: CNPq e Fundação de Apoio ao Ensino, Pesquisa e Assistência do Hospital das Clínicas da Faculdade Medicina de Ribeirão Preto (FMRP-USP)

Fisioterapeuta, Doutora em Biotecnologia pela Universidade Federal do Amazonas (UFAM) - Manaus (AM), Brasil.

Prof. Dr. School of Health and Life Sciences - New York Institute of Technology - New York, USA.

Profa. Dra. e Coordenadora-chefe do Curso de Biotecnologia da Universidade de Ribeirão Preto (UNAERP) - Ribeirão Preto (SP), Brasil

Prof. Dr. da Divisão de Dermatologia, Departamento de Clínica Médica e Centro Saúde Escola da Faculdade de Medicina de Ribeirão Preto da Universidade de São Paulo (FMRP-USP) - Ribeirão Preto (SP), Brasil

(C)2009 by Anais Brasileiros de Dermatologia
} 
estudos clínicos avaliaram a fototerapia no tratamento em vários tipos de úlceras crônicas, mas diferem quanto aos tipos e dosimetrias utilizados (comprimento de onda, potência, intensidade), que geram ceticismo da real eficácia na cicatrização tecidual. Recentemente, trabalhos realizados em nosso serviço, Caetano et al. ${ }^{6}$ (2009) demonstraram que a fototerapia acelera a cicatrização de úlceras venosas crônicas quando comparadas com curativo de sulfadiazina de prata $1 \%$ tópica ou terapia a luz placebo.

Assim, busca-se demonstrar o uso da fototerapia (LEDs 660/890nm) associada à sulfadiazina de prata tópica para a cicatrização de úlceras de perna em dois pacientes diabéticos.

\section{Casos clínicos}

Após assinatura do Termo de Consentimento Livre e Esclarecido (Processo HCFMRP/USP $\mathrm{n}^{\mathrm{O}}$ 302/06), dois pacientes diabéticos (tipo 2) selecionados aleatoriamente foram tratados no Ambulatório de Úlceras da Dermatologia do Centro Saúde EscolaFMRP-USP.

O aparelho de fototerapia (Dynatron Solaris ${ }^{\circledR}$ ) utilizado possuía a sonda 1-S1 (1 LED de 660nm, $5 \mathrm{~mW}$ ) e sonda 2 -S2 (32 LEDs de $890 \mathrm{~nm}$ e 4 LEDs de $660 \mathrm{~nm}, 500 \mathrm{~mW}$ ).

Após lavagem das úlceras com soro fisiológico $0,9 \%$, aplicou-se as sondas puntualmente sobre as feridas protegidas com PVC, na dose de $3 \mathrm{~J} / \mathrm{cm}^{2}$, $30 \mathrm{seg} / 5 \mathrm{~cm}^{2}, 2 \mathrm{x} / \mathrm{semana}$, seguido de curativos diários de sulfadiazina de prata a $1 \%$ (SDZ) creme por doze semanas de tratamento.

\section{RELATO DOS CASOS}

Caso 1: Paciente feminina, 77 anos, parda, diabética tipo 2, hipertensa e apresentando insuficiência venosa crônica (IVC), usuária de Metformina $(1700 \mathrm{mg} / \mathrm{d})$ e Captopril $(50 \mathrm{mg} / \mathrm{d})$. Apresentava hipertensão arterial sistêmica (HAS), cadeirante e úlceras de perna mistas há 25 anos, submetidas a vários tratamentos tópicos. Ao exame clínico-dermatológio, ambas as pernas apresentavam úlceras dolorosas, edema, dermatite ocre, lipodermatoesclerose e varizes. No membro inferior esquerdo (MIE), 2 úlceras (Figuras 1.1 e 1.2) foram tratadas com S1 e 4 no MID tratadas com S2 (Figuras 1.3 a 1.6) .

Caso 2: Paciente masculino, 50 anos, pardo, diabético do tipo 2 há 1 ano em insulinoterapia (22/10 UI dia), hipertenso há 20 anos, sequelado por AVC, cadeirante, com úlceras de perna múltiplas, dolorosas em ambas pernas, associadas a sinais de IVC. Ao exame, úlcera no MID há 1 mês (Figura 2.1) tratada com S1, e 3 úlceras no MIE com evolução de 10 meses (Figuras 2.2 a 2.4) tratadas com S2.

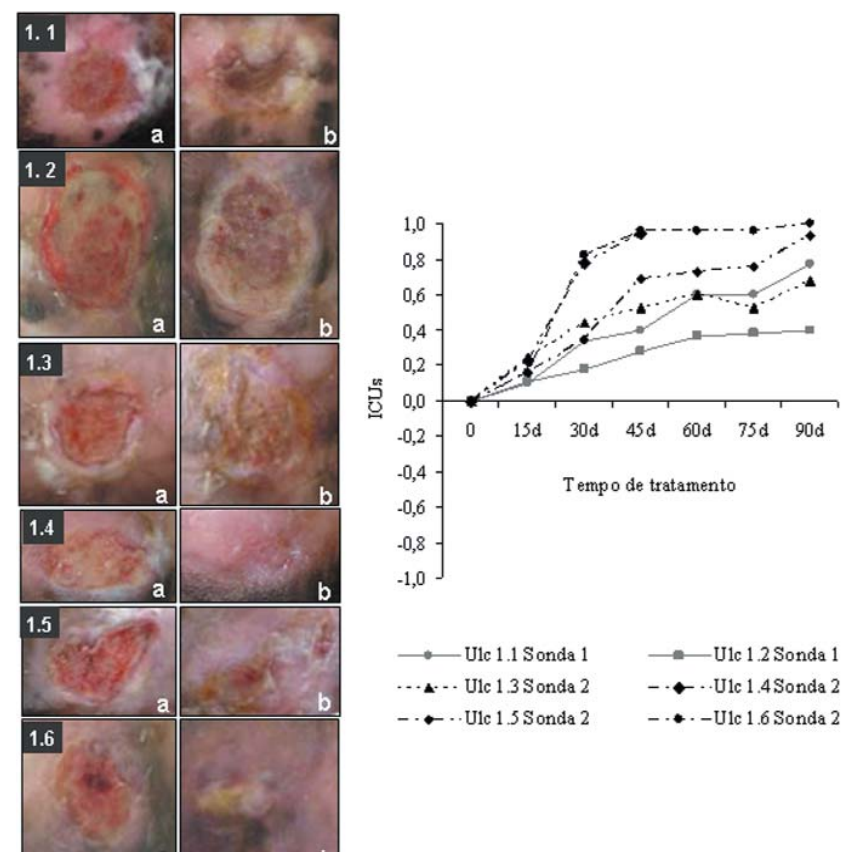

Figura 1: Aspectos clínico-evolutivos iniciais das úlceras de perna (a) e no $90^{\circ}$ dia (b). Respectivo gráfico da evolução quinzenal dos ICUs. Notam-se úlceras reepitelizadas totalmente na $7^{\mathrm{a}}$ e $11^{\mathrm{a}} \mathrm{sem}-$ ana de tratamento, respectivamente ( $1.4 \mathrm{~b}$ e $1.6 \mathrm{~b})$

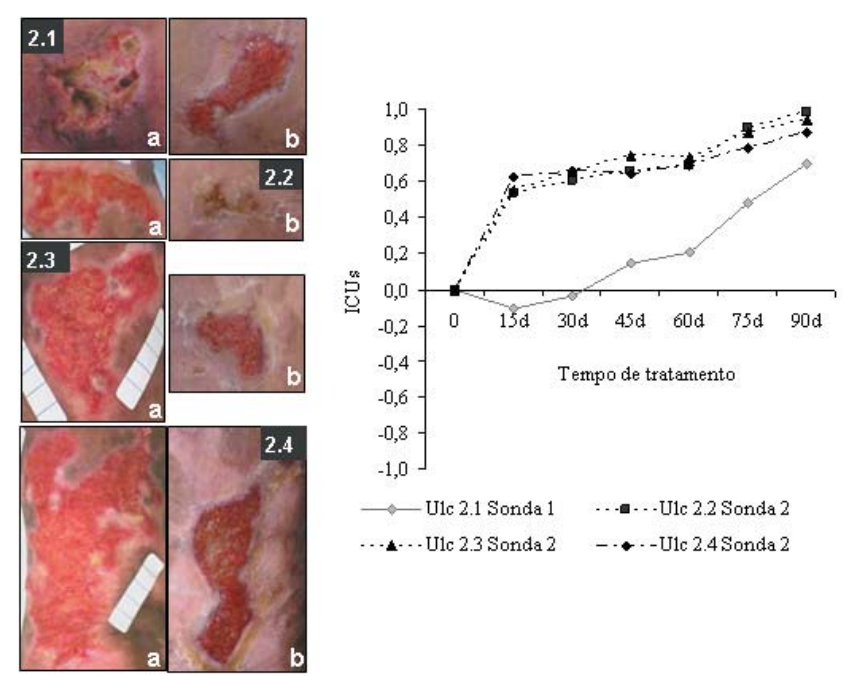

Figura 2: Aspectos clínico-evolutivos iniciais das úlceras de perna (a) e no $90^{\circ}$ dia de seguimento (b). Respectivo gráfico da evolução quinzenal dos ICUs

\section{Captura de análise das imagens das úlceras}

As úlceras foram avaliadas semanalmente por câmera digital (Sony ${ }^{\circledR}$ DSC-P100), fixada numa base de alumínio com régua milimetrada a $30 \mathrm{~cm}$ e perpendicular à úlcera. Pelo software Image $J^{\circledR}$ foi quantificada a área total das úlceras pela delimitação das bordas e calculado o índice de cicatrização das úlceras $[\mathrm{ICU}=($ Área inicial-Área final $) /$ Área inicial $)]^{7}$, onde $\mathrm{ICU}=1$ representa reepitelização total; $\mathrm{ICU}=0 \mathrm{sem}$ 
TABela1. Características das úlceras tratadas com sonda 1 e sulfadiazina de prata a $1 \%$ creme com suas respectivas evoluções clínicas representadas pelo índice de cicatrização das úlceras (ICU) e relação dos tecidos esfacelo e granulação $(\mathrm{E} / \mathrm{G})$

\begin{tabular}{|c|c|c|c|c|c|c|c|c|c|c|}
\hline \multirow[b]{2}{*}{$\begin{array}{l}\text { No. de caso. } \\
\text { ulcera }\end{array}$} & \multirow[b]{2}{*}{ Localização } & \multicolumn{3}{|l|}{ Tratamento } & \multicolumn{2}{|c|}{30 dias } & \multicolumn{2}{|c|}{60 dias } & \multicolumn{2}{|c|}{90 dias } \\
\hline & & $\begin{array}{l}\text { Tipo de } \\
\text { Tratamento }\end{array}$ & $\begin{array}{l}\text { Tempo } \\
\text { (sem) }\end{array}$ & $\begin{array}{l}\text { Área Inicial } \\
\left(\mathrm{cm}^{2}\right)\end{array}$ & ICU & E/G & ICU & $\mathbf{E} / \mathbf{G}$ & ICU & E/G \\
\hline 1.1 & $\begin{array}{l}\text { Maléolo } \\
\text { lateral MIE }\end{array}$ & $\mathrm{S} 1+\mathrm{SDZ}$ & 12 & 3 & 0,3 & 1,3 & 0,6 & 2,5 & 0,8 & 12,4 \\
\hline 1.2 & $\begin{array}{l}\text { Tendão de } \\
\text { calcâneo MID }\end{array}$ & $\mathrm{S} 1+\mathrm{SDZ}$ & 12 & 5,8 & 0,2 & 2,4 & 0,4 & 0,2 & 0,4 & 0,7 \\
\hline 2.1 & Face medial MID & $\mathrm{S} 1+\mathrm{SDZ}$ & 12 & $\begin{array}{l}11,5 \\
\text { Média }\end{array}$ & $\begin{array}{l}0,0 \\
0,2\end{array}$ & $\begin{array}{l}0,4 \\
1,4\end{array}$ & $\begin{array}{l}0,2 \\
0,4\end{array}$ & $\begin{array}{l}0,3 \\
1,0\end{array}$ & $\begin{array}{l}0,7 \\
0,6\end{array}$ & $\begin{array}{l}0,3 \\
4,4\end{array}$ \\
\hline
\end{tabular}

MID: Membro inferior direito; MIE: Membro inferior esquerdo; S1: Sonda 1; S2: Sonda 2; SDZ: Sulfadiazina de prata; E/G: Relação Esfacelo e Granulação; NC: Nada Consta

sinais de reepitelização; ICU $>0$ redução e $I C U<0$ aumento da área da úlcera.

Os tecidos presentes nas úlceras foram avaliados pela definição das cores através do plugin thresbold color do software Image ${ }^{\circledR}$, onde a cor vermelha correspondia ao tecido de granulação (G) e o amarelo, esfacelo/fibrina (E) (tecidos desvitalizados) ${ }^{8} \mathrm{O}$ dinamismo das modificações teciduais durante o tratamento foi estabelecido pela relação esfacelo/granulação (E/G) no momento da avaliação, onde $E / G=1$ mostra que a área de granulação é igual a esfacelo; $\mathrm{E} / \mathrm{G}<1$ mostra que a área de granulação é maior que esfacelo, ao contrário de $\mathrm{E} / \mathrm{G}>1$, quando granulação for menor que esfacelo.

\section{Análise dos resultados}

Nas tabelas 1 e 2 estão descritos a evolução clínico-terapêutica das áreas iniciais e os respectivos ICUs e $\mathrm{E} / \mathrm{Gs}$ no $30^{\circ}, 60^{\circ}$ e $90^{\circ}$ dias de tratamento com S1 e 2, respectivamente. As figuras 1 e 2 demonstram a evolução clínico-fotográfica inicial (a) e final (b) relacionado com suas respectivas evoluções quinzenais dos ICUs.

A análise evolutiva das úlceras com software Image $J^{\circledR}$ demonstrou que 6 úlceras tratadas com S2 apresentaram ICUs médios de 0,6; 0,7 e 0,9 (Tabela $2)$, enquanto 3 úlceras com $S 1$ tiveram valores médios de 0,$2 ; 0,4$ e 0,6 no $30^{\circ}, 60^{\circ}$ e $90^{\circ}$ dias (Tabela 1 ), respectivamente.

As úlceras 1.4 e 1.6 tratadas com a S2 apresentaram reepitelização total em 7 e 12 semanas de tratamento, respectivamente (Tabela 2 e Figura 1). Já as úlceras tratadas com S1 (Figuras 1.2 e 2.1), que apresentavam dor durante o tratamento, tiveram discretos sinais de cicatrização a partir da $5^{a}$ semana de tratamento (Figuras 1 e 2, Tabela 1) . A úlcera 1.2 que apre- sentou o ICU de 0,4 e E/G de 0,7 no $90^{a}$ dia devido a sua localização acima do tendão do calcâneo.

No entanto, em ambos os casos as úlceras tratadas com a S2 evoluíram com cicatrização satisfatória, seguido do relato de alívio da dor nas úlceras logo na $2^{a}$ semana de tratamento (Tabela 2 ).

\section{DISCUSSÃO}

O diabetes mellitus é uma síndrome multifatorial e crônica, que evolui com várias complicações desencadeadas pela macro e microangiopatia e/ou pela neuropatia, acometendo múltiplos órgãos como rim, retina, coração e pele. Essas complicações associadas às comorbidades como IVC e HAS, aumentam a possibilidade do surgimento de úlceras de perna. ${ }^{9}$ Além disso, os pacientes diabéticos apresentam suscetibilidade a traumas, infecções e, conseqüentemente às ulcerações, as quais se destacam pelo tratamento geralmente lento e de resultados limitados, o que diminui a qualidade de vida desses pacientes, podendo evoluir à amputação e óbito. ${ }^{10,11}$

A cicatrização das úlceras constitui-se de uma seqüência biológica complexa que envolve processos celulares e moleculares, como inflamação, formação tecidual (angiogênese, fibrogênese e reepitelização) e remodelagem tecidual. Clinicamente, as características teciduais das úlceras refletem a fase do processo cicatricial em que se encontra, como tecido necrótico ou amarelado (esfacelo) pela fase inflamatória inicial. A seguir, forma-se um tecido avermelhado e granulado (angiogênese), que se transforma num tecido de cor mais escura/vinhosa, compacto e sem aspecto granulado (fibroplasia). Por fim, a úlcera diminui sua superfície principalmente pela reepitelização das bordas e/ou ilhotas de reepitelização. Portanto, estes tecidos retratam o dinamismo da cicatrização da úlcera, 
TABela2. Características das úlceras tratadas com sonda 2 e sulfadiazina de prata a $1 \%$ creme com suas respectivas evoluções clínicas representadas pelo índice de cicatrização das úlceras (ICU) e relação dos tecidos esfacelo e granulação (E/G)

\begin{tabular}{|c|c|c|c|c|c|c|c|c|c|c|}
\hline \multirow[b]{2}{*}{$\begin{array}{l}\text { No. de caso } \\
\text { ulcera }\end{array}$} & \multirow[b]{2}{*}{ Localização } & \multicolumn{3}{|l|}{ Tratamento } & \multicolumn{2}{|c|}{30 dias } & \multicolumn{2}{|c|}{60 dias } & \multicolumn{2}{|c|}{90 dias } \\
\hline & & $\begin{array}{l}\text { Tipo de } \\
\text { Tratamento }\end{array}$ & $\begin{array}{l}\text { Tempo } \\
\text { (sem) }\end{array}$ & $\begin{array}{l}\text { Área Inicial } \\
\left(\mathrm{cm}^{2}\right)\end{array}$ & ICU & $\mathbf{E} / \mathbf{G}$ & ICU & $\mathbf{E} / \mathbf{G}$ & ICU & $\mathbf{E} / \mathbf{G}$ \\
\hline 1.3 & $\begin{array}{l}\text { Maléolo medial } \\
\text { MID }\end{array}$ & $\mathrm{S} 2+\mathrm{SDZ}$ & 12 & 2,5 & 0,4 & 1,0 & 0,6 & 0,3 & 0,7 & 3,0 \\
\hline 1.4 & $\begin{array}{l}\text { Face anterior } \\
\text { MID }\end{array}$ & $\mathrm{S} 2+\mathrm{SDZ}$ & 7 & 1,8 & 0,8 & 1,0 & 1,0 & 0,0 & 1,0 & $\mathrm{NC}$ \\
\hline 1.5 & $\begin{array}{l}\text { Face lateral } \\
\text { MID }\end{array}$ & $\mathrm{S} 2+\mathrm{SDZ}$ & 12 & 4,4 & 0,3 & 0,3 & 0,7 & 0,3 & 0,9 & 0,5 \\
\hline 1.6 & $\begin{array}{l}\text { Face póstero - } \\
\text { lateral MID }\end{array}$ & $\mathrm{S} 2+\mathrm{SDZ}$ & 12 & 4,4 & 0,8 & 0,3 & 1,0 & 1,0 & 1,0 & $\mathrm{NC}$ \\
\hline 2.2 & Face medial MIE & $\mathrm{S} 2+\mathrm{SDZ}$ & 12 & 14 & 0,6 & 0,2 & 0,7 & 0,4 & 1,0 & 0,0 \\
\hline 2.3 & Face anterior MIE & $\mathrm{S} 2+\mathrm{SDZ}$ & 12 & 26,3 & 0,7 & 0,3 & 0,7 & 0,8 & 0,9 & 0,1 \\
\hline \multirow[t]{2}{*}{2.4} & Face lateral MIE & $\mathrm{S} 2+\mathrm{SDZ}$ & 12 & 75,4 & 0,7 & 0,2 & 0,7 & 0,6 & 0,9 & 0,5 \\
\hline & & & & Média & 0,6 & 0,5 & 0,7 & 0,5 & 0,9 & 0,8 \\
\hline
\end{tabular}

MID: Membro inferior direito; MIE: Membro inferior esquerdo; S1: Sonda 1; S2: Sonda 2; SDZ: Sulfadiazina de prata; E/G: Relação Esfacelo e Granulação; NC: Nada Consta

que podem ser documentados percentualmente, representando o progresso ou deterioração da cicatrização através do tempo. ${ }^{8}$

Estudos clínicos mostram formas diferentes de avaliar e quantificar a evolução da cicatrização diante novas terapêuticas para úlceras crônicas, ${ }^{7,12}$ como pela variação da redução percentual da área das úlceras (AiAf/Ai)x100, que permite demonstrar a eficácia e comparar diferentes tratamentos, normalizando os diferentes tamanhos das úlceras, podendo ser utilizada em testes clínicos com pequena amostragem e de curta duração, além de indicar quantitativamente a continuidade ou modificação destas durante a evolução.

O software Image $J^{\circledR}$ utilizado permitiu a avaliação clínico-fotográfica das úlceras e a quantificação de suas áreas superficiais e as modificações teciduais durante o tratamento, o que permitiu demonstrar e quantificar a evolução dinâmica da cicatrização de úlceras cutâneas. 13

As úlceras tratadas com a S2 em ambos os casos apresentaram redução da área total maior que as úlceras tratadas com S1. Ressalta-se a localização da úlcera no caso da úlcera do tendão calcanear (úlcera 1.2) e evolução com menor ICU, atingindo ICU de 0,4 em 90 dias (Tabela 1 e Figura 1), provavelmente relacionada à menor vascularização local pela IVC, lipodermatoesclerose e inatividade física da paciente. Esse fato se confirma pela evolução da relação $\mathrm{E} / \mathrm{G}$ no $30^{\circ}$ dia que foi de 2,4 , indicando o aumento da inflamação, estabilizada no $60^{\circ}$ e $90^{\circ}$ dias, seguido de diminuição da área ulcerada.

Os resultados obtidos corroboram as evidências de que a fototerapia por meio de LEDs a 600-1000nm promove o reparo tecidual, particularmente nos casos de úlceras crônicas. ${ }^{2,3}$ Observa-se o efeito positivo da S2 para o tratamento de úlceras crônicas em pacientes diabéticos como observado por Kleinman et al., os quais constataram que 50 a $90 \%$ das úlceras diabéticas responderam positivamente à laserterapia com $785 \mathrm{~nm}$, assim como sua combinação com $632.8 \mathrm{~nm} .{ }^{14}$ Sugrue et al. também relataram resultados positivos do laser em vários tipos de feridas e úlceras, especialmente em casos crônicos e intratáveis. ${ }^{15}$ Nenhum efeito adverso foi informado em quaisquer destes estudos. Em parceria com New York Institute of Technology (USA), resultados semelhantes foram observados em nosso serviço com a fototerapia $(660 / 890 \mathrm{~nm})$ na cicatrização de úlceras venosas crônicas quando comparado à terapia a luz placebo e SDZ tópica diária. ${ }^{6}$

Os resultados sugerem que a associação de LEDs (660/890nm) duas vezes por semana com SDZ tópica diária mostrou maior eficácia na cicatrização quando comparado com S1 (1 LED) constituindo-se numa terapia bioestimuladora, não invasiva, de fácil e rápida aplicação com adicional efeito analgésico no tratamento de úlceras de perna em pacientes diabéticos, fazendo-se necessário a realização de estudos clínicos randomizados para consolidar nossos achados.

\section{AGRADECIMENTOS}

À Dynatronics pelo aparelho de fototerapia. Aos alunos Heriton Antonio e Thalis Silva pela colaboração no estudo. 


\section{REFERÊNCIAS}

1. DeFronzo RA, Ferrannini E. Insulin resistance. A multifaceted syndrome responsible for NIDDM, obesity, hypertension, dyslipidemia, and atherosclerotic cardiovascular disease. Diabetes Care. 1991;14:173-94.

2. Smith KC. Laser (and LED) therapy is phototherapy. Photomed Laser Surg. 2005;23:78-80.

3. Vladimirov YA, Osipov AN, Klebanov GI. Photobiological principles of therapeutic applications of laser radiation. Biochemistry. 2004;69:81-9.

4. Minatel DG, Frade MAC, França SC, Enwemeka CS. Phtototherapy promotes healing of chronic diabetic leg ulcers that failed to respond to other therapies. Lasers in Surgery and Medicine. 2009; DOI: 10.1002/ lsm. 20789.

5. Desmet KD, Paz DA, Corry JJ, Eells JT, Wong-Riley MT, Henry MM, et al. Clinical and experimental applications of NIR-LED photobiomodulation. Photomed Laser Surg. 2006;24:121-8.

6. Caetano KS, Frade MAC, Minatel DG, Santana LA, Enwemeka CS. Phototherapy improves healing of chronic venous ulcers. Photomed Laser Surg. 2009;27:111-8.

7. Robson MC, Hill D, Woodske ME, Steed DL. Wound healing trajectories as predictors of effectiveness of therapeutic agents. Arch Surg. 2000;135:773-7.

8. Fowler EM, Vesely N, Johnson V, Harwood J, Tran J, Amberry T. Wound care for persons with diabetes. Home Health Nurse. 2003;21:531-40.

9. Frade MAC, Cursi IB, Andrade FF, Soares SC, Ribeiro WS, Santos SV, et al. Úlcera de perna: um estudo de casos em Juiz de Fora-MG (Brasil) e região. An Bras Dermatol. 2005;80:41-6.

10. Pham H, Armstrong DG, Harvey C, Harkless LB, Giurini JM, Veves A. Screening techniques to identify people at high risk for diabetic foot ulceration: a prospective multicenter trial. Diabetes Care. 2000;23:606-11.

11. Frade MAC, Cursi IB, Andrade FF, Coutinho-Netto J, Barbetta FM, Foss NT. Management of diabetic skin wounds with a natural latex biomembrane. Med Cutan Iber Am. 2004;32:157-62.

12. Margolis DJ, Gelfand JM, Hoffstad O, Berlin JA. Surrogate end points for the treatment of diabetic neuropathic foot ulcers. Diabetes Care. 2003; 26: 1696-700.

13. Gomes FG, Santana LA, Minatel DG, Frade MAC. Uso do software Imaje $J^{\circledR}$ para análise clínico-fotográfica das úlceras. In: $5^{\circ}$ Encontro de Bioengenharia São Carlos. Caderno de Resumos do $5^{\circ}$ EncoBio. São Carlos: USP; 2005. p. 37.

14. Kleinman Y, Simmer S, Braksma Y, Morag B, Lichtenstein D. Low level laser therapy in patients with venous ulcers: Early and long term outcomes. Laser Ther. 1996;8:205-8.

15. Sugrue ME, Carolan J, Leen EJ. The use of infra-red laser therapy in the treatment of venous ulcerations. Ann Vasc Surg. 1990;4:179-81.

ENDEREÇO PARA CORRESPONDENNCIA / MAILING ADDRESS:

Prof. Dr. Marco Andrey Cipriani Frade

Divisão de Dermatologia da Faculdade de

Medicina de Ribeirão Preto - USP

Av. Bandeirantes 3900.

14048900 - Ribeirão Preto - SP

Tel./fax: 551636330236

e-mail: mandrey@fmrp.usp.br

Como citar este artigo/How to cite this article: Minatel DG, Enwemeka CS, FrançaSC, Frade MAC. Fototerapia (LEDs 660/890nm) no tratamento de úlceras de perna em pacientes diabéticos: estudo de caso. An Bras Dermatol. 2009;84(3):279-83. 